Zeitschrift f. Augenheilkunde 1899;2:1-4

\title{
Contents, Vol. 2, 1899
}

\section{Inhalts-Verzeichnis}

Originalapbeiten.

Cramer, E., Beitrag zu dem klinischen Verhalten intra-

oculârer Eisensplitter 96

Diumer, F., Ueber oberfläcliliclie gittrige Hornhaut-

trübung. (Mit 2 Abbildungen) 354

llaab, 0., Die gittrige Keratitis. (Hierzu Tafel Y) . . . 235 Hallauer, 0., Mucocele des Sinus frontalis. (Hierzu

2 Abbildungen)

Hedmaier, 0., Beitrag zur Frage des Zusanimeulianges

von Augen- und Nasenerkrankungen 540

Helbron, J., Beitrag zur Frage der Naplithalmwirkung auf

das Auge 433

Knapp, P., Experimentelle Untersucliungen über das. Ver-

fahren , künstliche Cataract zu erzeugen, ol·me die

vordere Kapsel zu zerreissen"553

Roster, W., Die Entwickelung der Ophtlialmologie in den

Niederlanden 109

Ruhnt, H. , Ueber Disticliiasis (congenita) vera. (Hierzu

Tafel III)

- $\quad$ Eine kurze Notiz zur Operation des Epicantbus . . 169

Markus, Cli. , Ein Fall von Conjunctivitis mit Rnötclien-

bildung, hervorgerufen (lurch eingedrungene Pflanzen-

L-aare. (Mit 3 Abbildungen) 34

Meyer, 0., Ein Fall von Schwangerscliaftsunterbrechung

bei líetinitis albuminurica gravidarum 34( \&gt;

Michel, J. v., Ueber Erkrankungen des Gefässsystems

der Arteria mid Vena centralis retinae mit besonderer

Berücksiclitigung der patliologiscb - anatomischeii Ver-

änderungen. (Hierzu Tafel I-11)

I

Peretti, Ein Fall von doppelseitiger gleichsinniger Linsen-

luxation nach Scliädelverletzung 225

Pergens, E., Ueber Vorgänge in der Netzliaut bei farbiger

Beleuclitung gleiclier Intensität 125

Peters, A., Bemerkungen über Erfolge der Nasenbehandlung bei Augenleiden 152

- $\quad$ Ueber autosuggerirte Myopie bei Seliulkindern . . 246

Pfalz, líeelle und eventuelle Unfallfolgen 516

Pines, L., Untersucliungen über den Ban der Retina mit 
Weigert's Neurogliamethode. (Hierzu Tafel V!) . . 252 líaelilmann, E., Ueber relativen und absoluten Mangel

des Farbensinnes. (Mit 5 Abbildungen) . . . 315, 403 Scliapringer, A., Die angcboreiie Sclüirze der Lidbindehaut,

eine bislier nocli niclit bescliriebene, typisclieMis,sbildung

des menscliliclien Auges. (Mit 9 Abbildungen), . 41

2

Sclieffels, 0., Ueber Bindehaii†transplantation zur Deckung

von Hornhautdefekten 427

Schwarz, 0., Zur „hemiopischen Pupillenakíion .... 533

Seydel, Zu den Girculationsstörungen der Netzhaut. (Hierzu

Tafel VII) " 349

Silex, P., Beitrag zur Kenntnis einiger seltener Gesichts-

anomalien. (Mit 10 Abbildungen) 141

Uhthoff, W., Ein Fall von einseitiger centraier Blendungs- $\bullet$ Retinitis durch elektrisches

Bo genlicht mit nachfolgender traumatischer Neurose. (Mit 1 Abbildung) . . . 341

Wintersteiner, II., Beitrag zur Kenntnis der Gebtirts*

verletzungen des Auges. (Mit 1 Tafel) 413

Sammelreferate.

Die Entsteliung der Stauungspapillitis. Von Prof. Dr. Karl

Baas in Freiburg i. B 170

Pathogenese und Therapie der spontanen Netzhautablösung.

Von Dr. Schrader in Gera ' 58

Die Behandlung der Erkrankungen cles Ihränen ableitenden

Apparates in der II. Iläífte des XIX. Jalirhunderts.

$\Lambda 7$ on Dr. Herm. Tödten in Hamburg .... 454, 561 Die Therapie des Ul·cas eorneae serpens von

Dr. Hugo

Wokenius 256

Berieht über die deutselie ophtlialmologische Litteratur.

I. Semester 1899:

I. Anatomie des Auges. Von Privatdoeent Dr.

J. Sobotta in Würzburg 276

II. Plivsiologie des Gesiehtssinnes. Von Privatdoeent

Dr. W. A. Nagel in Freiburg i. B 285

Mikroorganisineii. Von Dr. R. 0. Neumann in Würzburg 294

Pathologisehe Anatomie. Von Prof. v. Michel in Würzburg 361, 472

V. Angeborene Anomalien. Von Doc. Dr. Winter

steiner in Wien

557

Bericht über die amerikanische oplithalmologisclie Litteratur.

I. Semester 1899. Von Dr. Denig in New-York 379, 472 Bericht über die belgisehe Litteratur im Jahre 1898.

Von Dr. Ed. Pergens in Brüssel 188

Bericht über die belgisehe Litteratur im .lahre 1809.

I. Seni. Von Dr. Ed. Pergens in Brüssel .... 583 Bericlit über die holländische ophtlialmologische

Litteratur

des ,lahres 1898. Von G. J. Schoute in Amsterdam 
und Prof. Dr. W. Koster Gzn. in Leiden .... 203 Bericht über die holländische ophtlialmologische Litteratur

des Jahres 1899. I. Sem. Yob G. J. Schoute in

Amsterdam und Prof. Dr. W· Koster Gzn. in Leiden 589

$\cdot)$

Bericht über die polnische ophthalmologischeLitteratur. 1899.

I. $\quad$ Semester. Von Dr. v. Kamocki in Warschau . . 492

Bericlit über die ungarische ophtlmlmologische Litteratui $\cdot$

1899. I. Semester. Von Priv.-Doc. Dr. Emil v. G-rósz

in Budapest 298

Bericlit über die skandinavische ophthalinologisehe Litteratur.

1899. 1. Sem. Von Prof. W id mark in Stockholm . 580

Sitzung $\cdot$ sberiehte.

American o pli t b a 1 mo logical so c i ety :

Sitzung • vom 19. j'uli 1899 892,511

Bericht über die am 1. •luli 1899 in Düsseldorf abgebaltene

II. Versammlung rheinisch - westfälischer Augení”u $\%$,te. 219

Bericht über die ophthalmologische Abteilung der 71. Ver

sammlung Deutscher Naturforscher und Aerzte in

München. Von Dr. ü. v. Sicherer in München . . 498

Bericht über die Verhandlungen des IX. Iiiternationalen Ophth aim ologen- Congresses in

Utrecht. (14.-18. August 1899). Erstattet von Dr. 'Juni us, Prof. H. Kulint,

Dr. Pfalz Ergänzungsheft 1

Berliner medizin isc he Gesellschaft:

Sitzung vom 12. Juli 1899218

Be $r$ liner op lithalmologische $\mathrm{G}$ esellsc haft:

Sitzung vom 25. Mai 189990

„28. Juni 1899

„20. •luli $1899 \quad 218$

British medical association. Section of Ophthalmology:

67. Sitzung . .892

Chicago oph thalmo 1 ogi c a I society:

Februarsitzung 189988

Sitzung vom 14. März 189989

„11. April $1899 \quad 89$

Chicago ophth a liu ol ogi cal and otological society:

"Sitzung vom 9. Mai 1899 . . 215

Dor VII. Aerzte-C on gress zu Kasan vom 28. April bis

5. Mai d. J. .. ' 211

15. Versammlung des uiederländi sc hen augenärzt-lichen $\Lambda 7$ ereins:

Sitzung vom 7. Mai 1899215

Ophthal mological society of the united Kingdom:

Sitzung vom 9. Juni $1899 \quad$. . 210

„17. Juni $1899 \quad 216$

7. •luli $1899 \quad 217$

„12. Oktober $1899 \quad 599$ 
San Francisco Society of Eye, Ear, Nose and Throat Physicians:

Sitzung vom Februar 1899 . . 899

4 .

Section on Ophthalmology of tlfe New York Academy o f M e d i c i n e:

Sitzung vom 20. Februar $1899 \quad \cdots 90$

„20. März $1899 \quad 391$

„17. April $1899 \quad$ _. 597

Section on Ophthalmology, College of Physicians of Philadelph,ia:

Sitzung vom 21. Februar $1899 \quad 86$

„21. März $1899 \quad 87$

„18. April $1899 \quad 88$

Socie.tv of the Alumni of the city Hospital:

"Sitzung vom 12." April 1899 " 391

Société de médecine et de chirurgie de Bordeaux:

Sitzung vom 7. April 1899308

„21. April 1899 ........ 308

„26. April $1899 \quad 308$

Société des sciences médicales de Lyon:

Sitzung vom Januar $1899 \quad 307$

Société de médecine de Marseille:

Sitzung vom 5. Mai 1899309

Société d'ophthalmolo gie de Paris:

Sitzung vom 4. Juli 1899 .' 307

Société de médecine de Toulouse:

Sitzung vom 21. Februar 1899 308

„21. März 1899308

1. April 1899308

Société beige d'ophthalrn ologie:

Sitzung vom April $1899 \quad 213$

Thepapeutisehe Umschau.

iherapeutische Umschau 92, 222, 393, 518, 599

Unfall- und Saehverständig'enkunde.

Unfall- und Sachverstäiidigenkunde 96, 225, 516

Buehanzeig-en.

Maschke, M., Die augenärztliche Unfallpraxis .... 100

Tag esnachpiçhten.

iagesnachrichten $103,228,309,394$

Litteratur-Verzeiehnis.

Litteratur-Yerzeichnis .... 104, 228, 310, 395, 527, 602

Sach-Register . 609

Namen-lle \&lt; \&gt; ·ister 621

Zeitschrift für Augenheilkunde.

Unter ständiger Mitarbeit von

Dr. Denig-New-York, Prof. v. Ewetzki-Moskau, Prof. Falchi-Pavia,

Doc Di $\cdot$ v. Grósz-Budapest, Dr. Gruber-London, Dr. Kamocki-Warschau, Prof. Koster-Leiden, Dr. Ostwalt-Paris, Dr. Pergens-Brüssel. Prof. Widmark-Stockholm u, A., 
Doc. Dr. Bach-Würzburg, Prof. Czermak-Prag. Prot. Dimmer-Innsbruck, Prof. 0. Haab-Zürich. Prof. Kuhnt-Königsberg, Prof. Mellinger-Basel,

Prof. v. Michel-Würzburg, Prof. Hermann Pagensteeher- Wiesbaden. Prof. Peters-Bonn, Prof. Raehlmann-Dorpat. Prof. Schmidt-Rimpler-Göttingen, Prof. Silex-Berlin, Prof. Uhthoff-Breslau, Prof. Vossius- Giessen, Dr. Wilbrand-Hamburg, redigiert von

Prof. Dr. H. Kuhnt mi Prof. Dr. J von Michel

in $\mathrm{Kön} \mathrm{n}^{1} / 8$ sberg i. Pr. in Würzburg.

Band II

$\lambda$ lit vie]en Abbildungen im Text und 8 Tafeln.

Berlin 1899.

VERLAG VON S. KARGER.

KARLSTRASSE

$\neq \mathrm{v}^{\mathrm{o}} \%$

S. $\mathrm{K}$

T)

Alle Reclite vorbehalten.

í iedruckt bei Imberg \& Lefaon in Berlin SW. 